Result The mean score of quality of life of parents as a caregivers was in the test group $(224 / 9 \pm 24 / 11)$ and in the control group $(225 / 7 \pm$ 24/33). Quality of life for all samples before the intervention was unfavorable, but the mean scores in all dimensions of quality of life after 2 months of training in the experimental group $(7 / 86 \pm 338 / 2)$ and controls $(23 / 8 \pm 226 / 77)$ were changed. In the experimental group after training mean score of all samples increased. T-test analyzes confirmed these changes. $(\mathrm{P}<0 / 05)$. So the implementation of an educational intervention is effective in increasing family caregiver's quality of life.

\section{PEER COUNSELING OF ADOLESCENT HANDICAPPED CHILDREN AFTER SEX EDUCATION: THE PILOT STUDY}

doi:10.1136/archdischild-2012-302724.1857

H Soga, M Shirasaka, Y Komai, H Kuwata. Department of Clinical Nursing, Division of Pediatric Nursing, Shiga University of Medical Science, Otsu City, Japan

Background Most pediatric nurses recognize that the child with special needs requires the proper sex education, but we have avoided it so far, possibly unconsciously or just to avoid any unpredictable troubles.

Objective We have started to do the sex education for handicapped children.

Methods Three subjects had cerebral palsy and one subject had Werdnig Hoffmann disease. The contents of sex education were as follows; 1 . What's an adult?; 2. The situation of a fetus; 3 . Listening to their mother's talk about their childbirth; 4. Discussion about adolescent body image in a group. Children expressed their impressions on the sex education in the group composed of 4 children and 17 young volunteers. We recorded the contents of peer counseling and converted it into the text, and analyzed it by qualitative method.

Results We found 4 categories on the peer counseling. Four categories were 'Recognition of favorite persons of the opposite sex', 'Pleasure to talk about love story', 'Awareness of the differences in sex', 'Awakening to the respect for their parents'. Children understood about adolescent body image pretty well. They also understood how carefully they were brought up by their parents and consequently respected them very much.

Conclusion Although children could accept the sex education quite appropriately, the problem is that their experience on sex in future would be quite limited. The difficult theme which now confronts us is how to educate them about a tacit understanding, or sex behavior within the limited experience on the sex.

\section{PRESENTATION OF AN INDIVIDUALIZED WRITTEN INFORMATION SYSTEM TO IMPROVE KNOWLEDGE AND COPING AFTER CARDIAC SURGERY IN THE POSTNATAL PERIOD}

doi:10.1136/archdischild-2012-302724.1858

\section{E Hjorth-Johansen. Oslo University Hospital, Oslo, Norway}

After cardiac surgery in the postnatal period parents need to learn about their infant's congenital heart disease(CHD) and how to manage life after surgery. All information parents need is given in this stressful period and therefore might be difficult to remember and process. In Norway only Oslo University hospital(OUS) performs CHD surgery in the postnatal period and hence has the responsible for initial information to all parents in Norway.

Aims The main intended improvements has been to develop an individualized information system to increase knowledge and coping in parents and secure that all parents receive needed written and oral information before transfer to local health services. A secondary aim has been to improve the communication from OUS to local health services.
Method An interdisciplinary group from OUS children's department cooperated. After a thoroughly literature review medical knowledge and normalization of daily life was chosen to be highlighted. We individualized the information by dividing it into information categories like; pre- and postoperative considerations(2), different sirculatory consequences(5), medication management(12), nutrition, follow-up, a form to fill in about specific treatment and an uncompleted heart drawing to adapt to the infants diagnosis. A binder was designed to gather information and accompany infants trough coming years of follow-up and to be used in communication between parents and daycare and school. At discharge from OUS information sheets are distributed to local health service.

Results A nationwide and evidence based systematic information system that allows health personnel tindividualize informational needs and cooperate across health services.

\section{BUSINESS AS USUAL. A, PROVEN EFFECTIVE, MODEL TO TRAIN AND AUTHORISE NURSES IN RESERVED PROCEDURES IN A CHILDREN'S HOSPITAL}

doi:10.1136/archdischild-2012-302724.1859

'KA Unk, 'J Maaskant, 'E te Pas. 'Women's and Children's Clinic, Academic Medical Center, ${ }^{2}$ Department of Continuing Education, Academic Medical Center, University of Amsterdam, Amsterdam, The Netherlands

Background and Aims According to Dutch legislation nurses are allowed to perform a number of medical procedures, known as "reserved procedures". Nurses are permitted to perform these procedures, but only after thorough education, training, examination and authorisation. The aim was to develop and implement an efficient and effective model to facilitate these demands, on behalf of the (non-ICU) nursing staff of our children's hospital.

Methods We developed a cyclical blended learning method containing: e-learning, training of local assessors, bedside teaching, structural examination and registration.

The tool we developed is:

- feasible in daily practice,

- optimally using the local available skills and knowledge and

- based on the already present protocols.

\section{Results}

- We trained and authorised 30 local assessors.

- We trained, examined and authorised 215 nurses of 6 paediatric departments.

- We developed and used 8 e-learning modules (containing 13 procedures).

- We used this model in a 4-year cycle.

- We used a model which divides the set of reserved procedures into.

a. a general part (16 procedures) and

b. a ward-specific part (5-21 procedures).

Discussion/conclusion We successfully designed, introduced, implemented an effective and efficient intervention to ensure adequate nursing skills and knowledge regarding the "reserved procedures" in a children's hospital.

\section{PICU AND ADULT INTENSIVE CARE: THE ADVANTAGES OF A COMBINED TRAINING PROGRAM}

doi:10.1136/archdischild-2012-302724.1860

KV Lent. LUMC, Leiden, The Netherlands

Background and Aims In 1997 Pediatric intensive care unit united with adult IC. PICU has a 10-bed capacity. Since beginning of PICU in 1994 in LUMC, qualified nurses from the adult ICU had the opportunity to work in PICU. Some would keep working in PICU and train to qualify as Pediatric Critical Care Nurse. Since 2007 a 
new training program was introduced: new nursing students now have to qualify in adult ICU as well as in pediatric ICU. The aim is to evaluate this new training program.

Methods Using a written questionnaire we asked students for their motivation and experience regarding this new training program. We kept records of which nurses took care of which patient in each unit.

Results

- A sufficient inflow of young graduates.

- Sufficient qualified nurses in PICU

- $50 \%$ of who initially wanted to graduate in adult ICU nursing only, became enthusiastic about working in PICU and now continue to work in PICU either fulltime or parttime.

- Nurses from the adult units can come and help in PICU which means closure of beds could be prevented.

- The possibility of admitting an ill child at an adult unit when there is no bed available at PICU.

- Which very likely leads to less refusals.

Conclusion A good cooperation between adult and pediatric ICU can be very beneficial for patient care. Particularly, training ICU nurses in adult AND pediatric ICU leads to better staffing in PICU.

\section{EVALUATING THE EXPERIENCE OF USING ONLINE BLENDED LEARNING DURING INDUCTION PROGRAMIME FOR NURSES IN A LEVEL 3 NEONATAL UNIT}

doi:10.1136/archdischild-2012-302724.1861

BS Mathew. Neonatal, Homerton University Hospital City University, London, UK

Background and Aims The Department of Health has published guidances to help the NHS improve the care provided for premature and sick babies. However, a report by BLISS, the baby charity highlighted a shortage of specialist neonatal nurses within the UK. This often results in recruitment of in-experienced workforce who lack necessary knowledge and skills to perform safely in their workplace. This paper presents qualitative findings from a study which evaluated blended learning approach used in an induction programme for such novice nurses. The aim of the study was to explore registered nurses' experience on the use of blended learning.

Methods The study employed a mixed method approach and collected data from 20 newly recruited nurses using purposive sampling. Data were collected using questionnaires and semi structured interviews. Data analysis was by thematic content analysis.

Results The analysis of data revealed that nurses had a positive and satisfactory experience of using online blended learning which has enabled them to adapt to the new environment quickly. Most of them did not have previous online learning experience but wanted this to be implemented in future education programmes. The major barriers faced by the nurses participating in online learning were lack of time and motivational factors.

Conclusion Using social networking as a learning tool has a great potential in supporting nurses to develop their knowledge and skills. Advance planning and preparation are essential to enhance the quality of their learning experience.

\section{IMPLEMENTATION OF THE DUTCH GUIDELINE FOR THE CARE OF EXTREME PRETERMS: "THE LEARNING CURVE"}

doi:10.1136/archdischild-2012-302724.1862

JM Wielenga, A Claassen, R van der Hoek, S Broekman. IC Neonatology, Women's and Children Clinic Academic Medical Center Amsterdam, Amsterdam, The Netherlands

Background and Aims The Dutch multidisciplinary evidence based guideline on treatment decisions of infants born with a gestational age of at least 24 weeks was released in autumn 2010. The implementation of this guideline on the NICU of the Women's and
Children Clinic/Academic Medical Center in Amsterdam the Netherlands was a complicated process.

Methods A multidisciplinary workgroup, 5 nurses, 5 neonatologists and a pharmacist composed a phased implementation procedure which consisted of the following activities:

A multidisciplinary meeting before and 10 months after implementation; Existing protocols were made evidence based; Working visits were made; A centre of expertise was established; A group of experienced nurses and doctors were steered as leaders in the care; Clinics and workshops for leading nurses; Clinics for all professionals concerned; Sessions for nurses to reflect and learn from each other; Evaluation of the implementation; and an Inventory to readjust existing guideline.

Results Due to media attention, an early start was forced. Protocols were not ready, material and equipment not available. The number of infants was higher than expected. The decision to perform the care for these infants by leading nurses resulted in agitation amongst other nurses. Their opinion was that complex nursing care should be performed by all experienced nurses. New rules resulted in a lack of clarity.

Conclusions Well-considered choices were made, however it appeared that nurses were in need of training on the job instead of planned theoretical training in advance.

\section{STUDYING THE HOSPITALIZATION CAUSES OF NEONATES FROM FARS PROVINCE AT THE NURSERY WARDS}

doi:10.1136/archdischild-2012-302724.1863

'M Edraki, ${ }^{2} \mathrm{H}$ Morravej, ${ }^{2 M}$ Rambod, ${ }^{2} \mathrm{~S}$ Montaseri. 'Shiraz University of Medical Sciences; ${ }^{2}$ Nursing and Midwifery, Shiraz University of Medical Sciences, Shiraz, Iran

Introduction and objective Immature neonates are exposed to be affected by Infectious, Pulmonary, Cardio-Vascular, Digestive, Metabolic and Endocrine diseases. The aim of present study is to survey the hospitalization causes of immature neonates at thenursery wards.

Materials and Methods This study is a descriptive research. The samples were consisting of 60 immature neonates less than 37 weeks and weight of below 2500 grams who had been hospitalized at the nursery wards of Shiraz city. These neonates were selected by simple sampling method.

Results The results of the study showed that, $53.5 \%$ of hospitalization causes of immature neonates at the hospital were affection to the Jaundice and Respiratory Distress Syndrome followed by Pneumonia which was the cause of $30.0 \%$ of cases. While, affection to the diseases like Sepsis, Hypoglycemia, Necrosing Enterocolitis, Metabolic disorders and Nosocomial infection were the cause of $8.3 \%, 5.0 \%, 3.3 \%, 1.7 \%$ and $1.7 \%$ of the hospitalization cases respectively. None of them were hospitalized due to the intra cerebral hemorrhage in this study.

Discussion and conclusion In this study, Jaundice, RDS and Pneumonia are among the most prevalent causes of hospitalization of immature neonates in the hospital.

It is recommended for the health care givers to have sufficient information in this regard and while facing immature neonates, pay more attention to their respiratory condition.

\section{EXPERIENCES OF PARENTS OF CHILDREN WITH CANCER: A BASIS FOR THE TRAINING OF NURSES}

doi:10.1136/archdischild-2012-302724.1864

Z Molazem, Z Yazdanpanahi, H Reisi. School of Nursing \& Midwifery Shiraz University of Medical Sciences and Health Services, Shiraz, Iran

Background and Aims Being confronted with the diagnosis of childhood cancer in the family causes various emotional reactions. 